\title{
Induction and Experience-Dependent Consolidation of Stable Long-Term Potentiation Lasting Months in the Hippocampus
}

\author{
Wickliffe C. Abraham, ${ }^{1}$ Barbara Logan, ${ }^{1}$ Jeffrey M. Greenwood, ${ }^{2}$ and Michael Dragunow ${ }^{2}$ \\ ${ }^{1}$ Department of Psychology, University of Otago, Dunedin, New Zealand, and '2Department of Pharmacology, University of \\ Auckland Medical School, Auckland, New Zealand
}

Long-term potentiation (LTP) is widely regarded as a memory mechanism, but it is not known whether it can last long enough to underlie very long-term memory. We report that highfrequency stimulation (HFS) paradigms applied to the rat dentate gyrus can elicit stable LTP lasting months and up to at least 1 year. The induction of stable LTP was sensitive to stimulation variables on the day of HFS and was associated with phosphorylation of CAMP response element-binding protein. The maintenance of stable LTP was also experience-dependent, because it was reversed when animals were exposed repeatedly to an enriched environment beginning $14 \mathrm{~d}$ post-HFS.
However, stable LTP eventually consolidated over time and became resistant to reversal, because exposure to enriched environments $90 \mathrm{~d}$ post-HFS failed to influence stable LTP maintenance. Thus, LTP can be shown to meet one of the principal criteria for a very long-term memory storage mechanism. However, under naturalistic environmental conditions, LTP may normally be retained in the hippocampus for only short periods of time.

Key words: long-term potentiation; memory; hippocampus; enriched environment; CREB; depotentiation; NMDA receptor
Long-term potentiation (LTP) of synaptic efficacy is widely regarded as a putative long-term memory mechanism. This is based in part on the reported persistence of LTP over time in freely behaving animals. Evidence to date, however, indicates that although LTP in the dentate gyrus can last from a few days to many weeks, it does not persist stably enough to support very long-term memories lasting months or longer (Racine et al., 1983; Abraham and Otani, 1991). Although these findings are consistent with theoretical and empirical evidence that the hippocampus functions as a temporary memory store (Marr, 1971; Milner, 1989; Squire, 1992), there is growing evidence that, in fact, the hippocampus may have the capacity for more permanent information storage (Nadel and Moscovitch, 1997). The question remains, therefore, whether LTP in the hippocampus is necessarily decremental or whether it can be maintained stably under some conditions. Although it has been shown previously that theta-burst stimulation in area CA1 can induce LTP that is stably maintained over several weeks (Staubli and Lynch, 1987), it cannot be determined from these data whether the LTP would have been maintained over months.

A complicating factor in the investigation of the inherent stability of induced LTP is its sensitivity to behavioral variables after the period of induction. In two studies, it has been shown that exposure to a mildly stressful novel environment (Xu et al., 1998) or novel stimulus (Manahan-Vaughan and Braunewell, 1999) shortly after the induction of LTP in CA1 will cause a rapid and

\footnotetext{
Received June 14, 2002; revised Aug. 8, 2002; accepted Aug. 22, 2002.

This research was supported by grants from the New Zealand Health Research Council and the New Zealand Marsden Fund. We thank Assoc. Prof. D. Bilkey, Dr. D. Ireland, Dr. B. Mockett, Assoc. Prof. J. Wickens, and Dr. A. Heynen for comments on previous versions of this manuscript, and P. Curtis and S. O'Carroll for excellent technical assistance.

Correspondence should be addressed to Prof. Wickliffe C. Abraham, Department of Psychology, University of Otago, Box 56, Dunedin, New Zealand. E-mail: cabraham@psy.otago.ac.nz.

Copyright (c) 2002 Society for Neuroscience $\quad 0270-6474 / 02 / 229626-\bullet \$ 15.00 / 0$
}

persistent reversal of the LTP. Interestingly, the novel experiences were effective at reversing LTP when given $1 \mathrm{hr}$, but not 24 $\mathrm{hr}$, after induction, indicating that the experiences interfered with the initial consolidation but not the maintenance of LTP. It is not clear from these studies, however, whether the novel experience was itself sufficient to reverse LTP, or whether the low-frequency stimulation given simultaneously was also required.

We have revisited the issue of LTP persistence in the dentate gyrus region of the hippocampus and found that LTP can indeed show longevity appropriate for a mechanism underlying memory storage lasting months or longer. We also found that such stable LTP is nonetheless profoundly sensitive to experience and can be reversed after exposure to enriched environments in the weeks after LTP induction. However, stable LTP appeared to eventually consolidate and become resistant to experience-induced decay.

Portions of these data have been published previously in preliminary form (Abraham et al., 2001).

\section{MATERIALS AND METHODS}

Surgery. Adult male Sprague Dawley rats (350-500 gm) were anesthetized with sodium pentobarbital $(60 \mathrm{mg} / \mathrm{kg}$, i.p.). Following standard stereotaxic surgical procedures, stimulating and recording stainless steel wire electrodes, insulated except for the cut tip, were implanted bilaterally to establish perforant path-evoked field potentials recorded in the dentate hilus, as described previously (Abraham et al., 1993). The initial slope of the field EPSPs (fEPSPs) was used as the measure of synaptic efficacy. After surgery, animals were housed individually in small standard cages and exposed to a normal $12 \mathrm{hr}$ light/dark cycle.

Electrophysiology. Beginning 2 weeks after surgery, animals were taken to a recording room and tested for usable recordings (fEPSP slope $\geq 3.5$ $\mathrm{mV} / \mathrm{msec}$ at stimulus currents $\leq 500 \mu \mathrm{A}$ ). If the recordings met these criteria, baseline testing $(0.05-0.017 \mathrm{~Hz}, 150 \mu \mathrm{sec}$ pulse duration, alternating between the two hemispheres for 20-30 min) was undertaken, using a stimulus strength that elicited a $2-4 \mathrm{mV}$ population spike. Baseline recordings were made at the same time of day (during the animal's light cycle) two to three times per week until a stable level of evoked responses was obtained for at least four consecutive sessions, i.e., the responses varied by less than $\pm 5 \%$. The responses from these sessions were then used to calculate an average baseline response. On the day of 
LTP induction, high-frequency stimulation (HFS) was delivered after a $30 \mathrm{~min}$ baseline recording period, and responses were followed for another $60 \mathrm{~min}$. Recording sessions were generally conducted on days 1 , 2, 3, 4, and 7 after tetanus (or else days 1,3,5, and 7 after tetanus) and two times per week thereafter. The level of LTP on these days was calculated as the percentage fEPSP increase (averaged over the last half of the recording session when responses are stable) relative to the baseline average response, as is our standard procedure (Abraham et al., 1993, 1994). The responses early in a recording session are somewhat variable because of the effects of handling and animal transfer to the recording chamber. LTP on the day of tetanization, however, was calculated relative to the average fEPSP recorded over the $15 \mathrm{~min}$ before tetanus. Similarly, the percentage changes for the control nontetanized hemisphere fEPSPs were calculated relative to their own average baseline value.

HFS protocols. On the day of tetanization, HFS was applied to the perforant path in one hemisphere using $400 \mathrm{~Hz}, 25 \mathrm{msec}$ trains (at the baseline stimulus current intensity but with the pulse duration increased to $250 \mu \mathrm{sec}$ ). The nontetanized hemisphere served as a control for changes in an animal's arousal and hormonal state, and experiences over time, age, activity levels, etc. Trains were delivered in sets of five trains, with $1 \mathrm{sec}$ between trains and either 1 or $10 \mathrm{~min}$ between sets. The number of sets of HFS trains delivered per animal varied across groups. The hippocampal electroencephalogram was displayed on an oscilloscope during HFS to monitor for epileptiform afterdischarges, but none were observed in this study (although such abnormal activity is often seen after HFS given to area CA1, indicating that this method is capable of detecting afterdischarges.)

In four animals, stimulating electrodes were placed separately in the medial and lateral perforant pathways according to previous protocols (Abraham et al., 1994). Baseline recordings were made for the lateral path before and after HFS as described above. During HFS, nearsimultaneous tetanization was delivered to both the medial and lateral perforant paths, with each lateral path stimulus preceding each medial path stimulus by $5 \mathrm{msec}$. No control hemisphere recordings were made in these animals for technical reasons.

Data analysis. LTP was defined as an fEPSP increase $\geq 15 \%$ measured 60 min post-HFS. In animals in which the criterion was not met, the LTP was classified as decremental; in those in which the criterion was met, LTP maintenance was analyzed further. First, the fEPSP measurements made in the tetanized hemisphere were adjusted for variations in the recordings of the control hemisphere, where available, by subtracting the percentage changes in the control hemisphere fEPSPs from the those in the tetanized hemispheres. Single three-parameter negative exponential curves were then fitted to the LTP maintenance data, beginning on day 1 post-HFS, without being constrained as to the final baseline level or the slope of the function. The equation used was $y=y_{0}+a e^{(-\mathrm{bt})}$, where $y_{0}$ is the exponential asymptote, $a$ is a value that gives the $y$ intercept when added to the asymptote, $b$ is the rate of exponential decay, and $t$ is the time after LTP induction in days. The reported decay time constant $(\tau)$ is the inverse of the decay rate parameter $b$ and is the time taken for the function to decline by $63 \%$. Stable LTP was defined as a dataset for which the exponential asymptote was $\geq 10 \%$ above baseline, and the recordings were maintained for at least $42 \mathrm{~d}$ after tetanus. It should be noted that only $\sim 50 \%$ of the animals had usable recordings in the control hemisphere. However, stable LTP was identifiable in both raw and corrected datasets, indicating that correction for control recordings was not necessary for its identification. A few animals showed marked, precipitous changes in the recordings from one or both hemispheres beginning at random times after tetanus. In these cases, the wave-shapes also changed in a way characteristic of a significant movement of the recording electrode position, such as the development of a late negativity in the waveform or even a complete reversal of the positive-going waveform into a negative-going waveform. Where such events occurred in the tetanized hemisphere, the exponential asymptotes for the tetanized hemispheres in these animals were more negative than $-50 \%$. Accordingly, all data from these animals $(n=4)$ were discarded, unless specified otherwise.

Immunohistochemistry and densitometry. Animals were killed by overdose with halothane at defined times after tetanization, and the brains were removed, frozen on dry ice, and stored at $-70^{\circ}$. Coronal sections $(16 \mu \mathrm{m})$ through the dorsal hippocampus were thaw-mounted onto poly-L-lysine-coated glass slides. Immunohistochemical staining with an antibody to serine-133-phospho-cAMP response element-binding protein (pCREB) (UBI; 1:500 dilution) was performed as described previ- ously (Butterworth and Dragunow, 1996), with minor changes. Endogenous peroxidase activity was blocked by incubation in $\mathrm{H}_{2} \mathrm{O}_{2}(1 \%$ in $50 \%$ methanol, $5 \mathrm{~min}$ ), and all PBS solutions contained $0.2 \%$ Triton X-100. Densitometric analysis was performed on blinded sections at $125 \times$ magnification using MD30plus software (Leading Edge). For each section, staining densities for the entire dentate granule cell layer were normalized to background staining, which was measured bilaterally in the stratum radiatum in a camera field ( $1 \mathrm{~mm}$ width) immediately medial to a vertical line through the lateral end of the dentate gyrus lower blade. Densitometry measurements were corrected for light source and camera drift. Data were pooled for two to three sections from each brain.

Enriched environment. Animals in the enriched environment (EE) group were housed individually in standard cages but periodically were placed in groups of two to three in a large $(70 \times 100 \times 40 \mathrm{~cm})$ fiberglass box containing novel objects, drinking water, normal lab chow, and Kellogg's Cocoa Pops scattered throughout the chamber. The box was in a room different from the vivarium and the recording room. The arrangement of objects was varied daily. The rats were placed in the enriched environment for either $1 \mathrm{hr} / \mathrm{d}$ for $21 \mathrm{~d}$ during the light cycle or overnight for $14 \mathrm{hr}$ (including the entire $12 \mathrm{hr}$ dark cycle) for $7 \mathrm{~d}$. When electrophysiological recordings were made, these occurred 3-4 hr before placement in the enriched environment for that day. Animals in the home cage control group (HC) remained unhandled in the vivarium, except during electrophysiological recordings and routine cage cleaning. Exposure to the enriched environment generally had little effect on fEPSPs in the control hemisphere, although transient elevations of the population spike were noted during the period of EE treatment (Irvine and Abraham, 2001). Thus exposure to EE by itself did not produce any net LTP or long-term depression (LTD) effects, although EE may have caused LTP and LTD at different synapses such that there was no net change in overall synaptic efficacy, as detected by field potential recordings.

\section{RESULTS}

\section{Stable LTP can be induced in the dentate gyrus, depending on the stimulus protocol}

The experiments were conducted while the rats were quietly awake. Before tetanization, there were no significant differences between HFS groups in either the baseline EPSP slope or population spike amplitude (one-way ANOVA; $p>0.4$ ) (Table 1). When five trains of HFS (5T) were administered, a moderate LTP was induced on average $(15 \pm 5 \% ; n=6)($ Fig. $1 A)$. Two animals did not reach LTP criterion ( $\geq 15 \%$ change), and in the remaining four cases the LTP decayed rapidly to baseline levels within $48 \mathrm{hr}$ (mean $\tau=0.7 \mathrm{~d}$ ). Twenty trains of HFS (20T) induced more robust LTP $(30 \pm 3 \% ; n=6)$ (Fig. 1A). In accord with our previous findings (Abraham et al., 1993), this LTP also decayed to baseline for five of six animals, with an average decay time constant of $1.0 \mathrm{~d}$ as determined by fitted exponential functions. For one animal, however, the LTP did not decay but stabilized unexpectedly at an asymptotic level of $23 \%$ LTP that lasted at least $77 \mathrm{~d}$, the longest it was tested. To formally categorize such stable LTP, we adopted the stringent criteria that the LTP must have been recorded for at least $42 \mathrm{~d}$ post-HFS and that the asymptote of the negative exponential function fitted to the

\begin{tabular}{|c|c|c|c|c|}
\hline $\begin{array}{l}\text { HFS } \\
\text { protocol }\end{array}$ & $\begin{array}{l}\text { Days } \\
\text { between } \\
\text { surgery } \\
\text { and HFS }\end{array}$ & $\begin{array}{l}\text { Recording } \\
\text { sessions } \\
\text { before } \\
\text { HFS }\end{array}$ & $\begin{array}{l}\text { Baseline } \\
\text { fEPSP } \\
(\mathrm{mV} / \\
\mathrm{msec})\end{array}$ & $\begin{array}{l}\text { Baseline } \\
\text { PS (mV) }\end{array}$ \\
\hline $5 \mathrm{~T}$ & $40 \pm 7$ & $5.3 \pm 0.2$ & $5.7 \pm 1.0$ & $3.0 \pm 0.5$ \\
\hline $20 \mathrm{~T}$ & $39 \pm 3$ & $7.4 \pm 0.7^{*}$ & $6.2 \pm 0.5$ & $3.0 \pm 0.4$ \\
\hline $4 * 5 \mathrm{~T}$ & $43 \pm 3$ & $7.8 \pm 0.6^{*}$ & $5.1 \pm 0.5$ & $3.0 \pm 0.3$ \\
\hline $50 \mathrm{~T}$ & $47 \pm 4$ & $6.4 \pm 0.5$ & $5.1 \pm 0.3$ & $2.5 \pm 0.4$ \\
\hline
\end{tabular}

*Significantly different from 5T group; $p<0.05$; post hoc Student's-Newman-Keuls test. PS, Population spike. All values are mean \pm SEM 
Figure 1. Protocol dependence of stable LTP in the dentate gyrus. $A$, LTP induction by different $400 \mathrm{~Hz}$ HFS protocols. All protocols induced a significant LTP $(p<0.05)$, measured 55-60 min post-HFS, that was not statistically significantly different between groups (one-way ANOVA; $p>0.1$ ). 5T, 20T, and 50T indicate the total number of $400 \mathrm{~Hz}$ tetanic trains, delivered as sets of five trains, 1 min apart. $4 * 5 T$, Four sets of five trains delivered $10 \mathrm{~min}$ apart. Data are mean \pm SEM. $B$, Summary histogram illustrating the percentage of animals showing stable LTP in the dentate gyrus for each protocol used. Both the pattern and number of stimulus trains affected the probability of stable LTP occurrence. 5T, Zero of 6 animals; 20T, 1 of $6 ; 4 * 5 T$, 3 of $6 ; 50 T, 8$ of 12. C, Decremental LTP for one animal given $4 * 5 \mathrm{~T}$ (arrow). Data for both tetanized $(\mathbf{O})$ and control $(\bigcirc)$ pathways are plotted. Average baseline value is represented by the dotted line. Insets are the rising phases of the fEPSPs (averages of 10 responses) before population spike onset and recorded from the tetanized hemisphere at the times indicated relative to HFS. Calibration: $2 \mathrm{mV}, 0.5 \mathrm{msec}$. $D$, Stable LTP for one animal given 50T. Data for both tetanized $(\bullet)$ and control $(\bigcirc)$ pathways are plotted. Inset waveforms as in $C$. The data for the tetanized hemispheres in $C$ and $D$ have not been corrected for the control hemisphere changes.
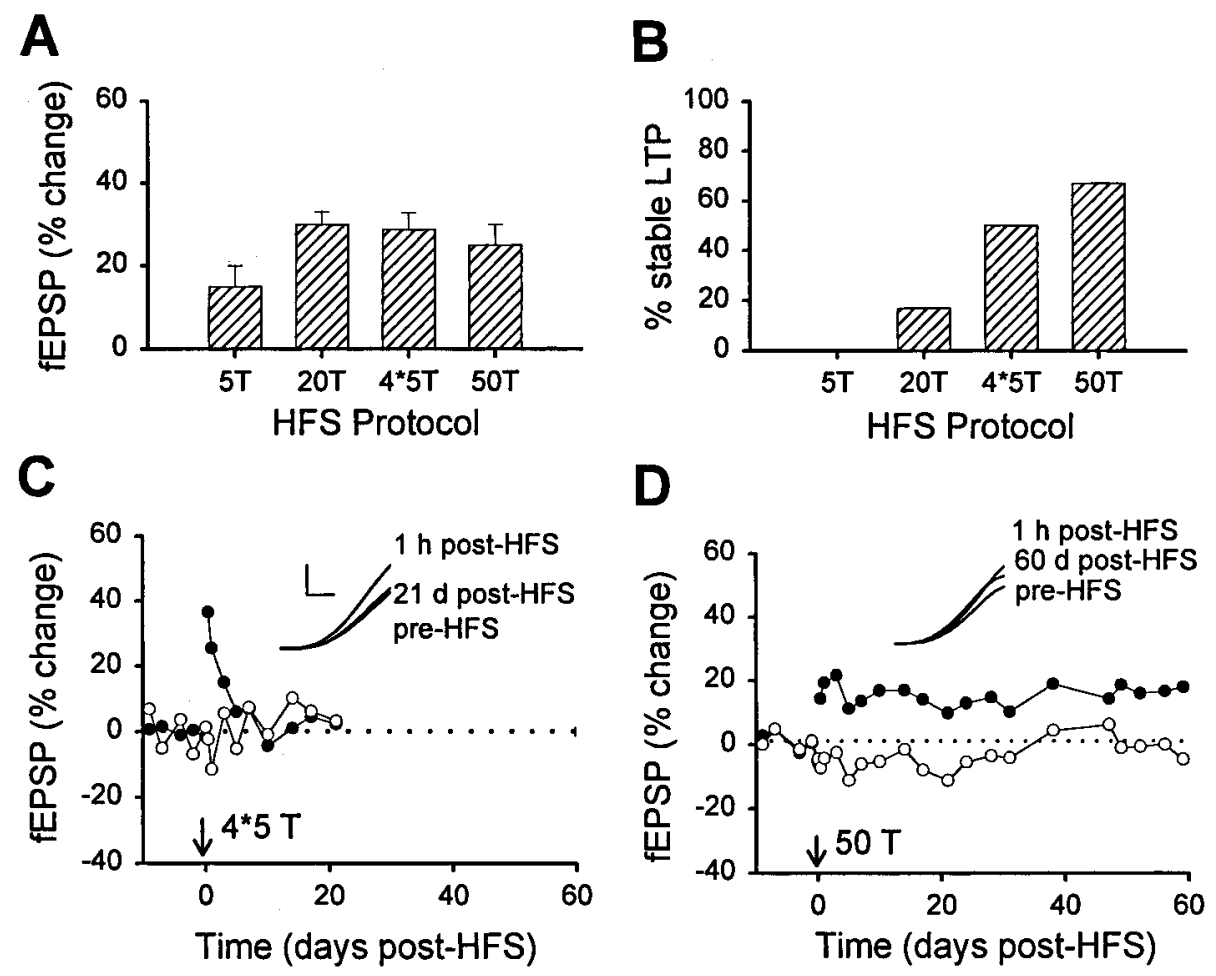

post-HFS LTP values was $\geq 10 \%$ above baseline. LTP that did not meet these criteria for stability was classified as decremental.

To find more reliable methods of generating stable LTP in the dentate gyrus, two additional tetanization paradigms were used. First, the 20T HFS was altered by separating the four sets of five trains by $10 \mathrm{~min}(4 * 5 \mathrm{~T})$, instead of the standard $1 \mathrm{~min}$, because previous studies have shown that the delivery of stimulus trains spaced over time can prolong the duration of synaptic plasticity (Huang and Kandel, 1994). Using this protocol, robust LTP was again obtained $(29 \pm 4 \% ; n=6)$ (Fig. $1 A)$, and three of six animals met the criteria for stable LTP (Fig. $1 B$ ), with an average asymptotic level of $24 \%$ LTP. The remaining three animals showed rapid LTP decay, with an average time constant of $3.3 \mathrm{~d}$ (Fig. 1C). In the second paradigm, 50 trains (50T) were delivered using our standard pattern of massed trains ( 1 min between sets of five trains). Once again, robust LTP was generally induced (25 $\pm 5 \% ; n=12$ ) (Fig. $1 A)$, although in this condition three animals showed $<15 \%$ initial potentiation. Remarkably, however, 8 of 12 animals met the criteria for stable LTP, with an average asymptote of $20 \%$ LTP (Fig. $1 B, D$ ).

The frequency of stable LTP occurrence varied significantly between the four HFS groups $\left(\chi^{2}=9.17 ; p<0.05\right)$ (Fig. $\left.1 B\right)$. This suggests that the apparent stability of LTP was not caused by a random upward instability of the responses but rather by the pattern and number of stimulus trains used to induce the LTP. However, it is important to consider whether the apparently stable LTP was caused by some aspect of our experimental or analysis procedures. First, it is possible that these data were biased by the fact that the data for four animals were discarded because of major response changes that developed at a random time after tetanus in one or both hemispheres. However, these four animals were relatively evenly represented across the groups (20T, $n=1$; $4 * 5 \mathrm{~T}, n=2 ; 50 \mathrm{~T}, n=1)$. Furthermore, even if these animals were categorized as showing decremental LTP and included in the $\chi^{2}$ analysis, there was still a significant difference in the occurrence of stable LTP across groups $\left(\chi^{2}=8.87 ; p<0.05\right)$. Second, it is possible that the procedure of correcting for control hemisphere changes could have made a decremental LTP appear as stable LTP, if both the control and potentiated responses were gradually declining equally over time. To assess this possibility, exponential fits were applied only to the data from the tetanized hemispheres to assess LTP stability. This resulted in changing the categorization of four animals: LTP in two animals changed from stable to decremental (one each in the $4 * 5 \mathrm{~T}$ and $20 \mathrm{~T}$ groups), and the reverse was true in two other animals (1 each in the $4 * 5 \mathrm{~T}$ and $20 \mathrm{~T}$ groups). This left the frequency of stable LTP across groups unchanged, and thus $\chi^{2}$ analysis continued to show a significant difference between groups. Finally, we considered whether the number of days or baseline recording sessions between surgery and tetanization could account for the group differences in the stability of LTP. A one-way ANOVA revealed no significant difference between groups in the time between surgery and tetanus $(p>0.4)$ (Table 1$)$. In contrast, there was a significant group difference in the number of baseline recording sessions before tetanus $\left(F_{(3,30)}=3.14 ; p<0.05\right)$. However, this was not related to LTP stability because post hoc Student's-Newman-Keuls pairwise comparisons revealed that the only significant differences were between the $5 \mathrm{~T}$ group on the one hand and the $20 \mathrm{~T}$ and $4 * 5 \mathrm{~T}$ groups on the other (Table 1 ). The $50 \mathrm{~T}$ group, with the highest frequency of stable LTP occurrence, did not differ on this measure from any of the other three groups. Taking all of these additional analyses together, we conclude that LTP stability was not an artifact of discarding animals from the study, the amount of handling and recording before tetanus, variations in electrophysiological parameters before tetanus, or the use of control hemisphere data for correction purposes.

To compare the temporal profile of maintenance between stable and decremental LTP, the data from animals receiving 20 or more trains were pooled according to their LTP classification (Fig. 2A). For this comparison, we also selected only those 

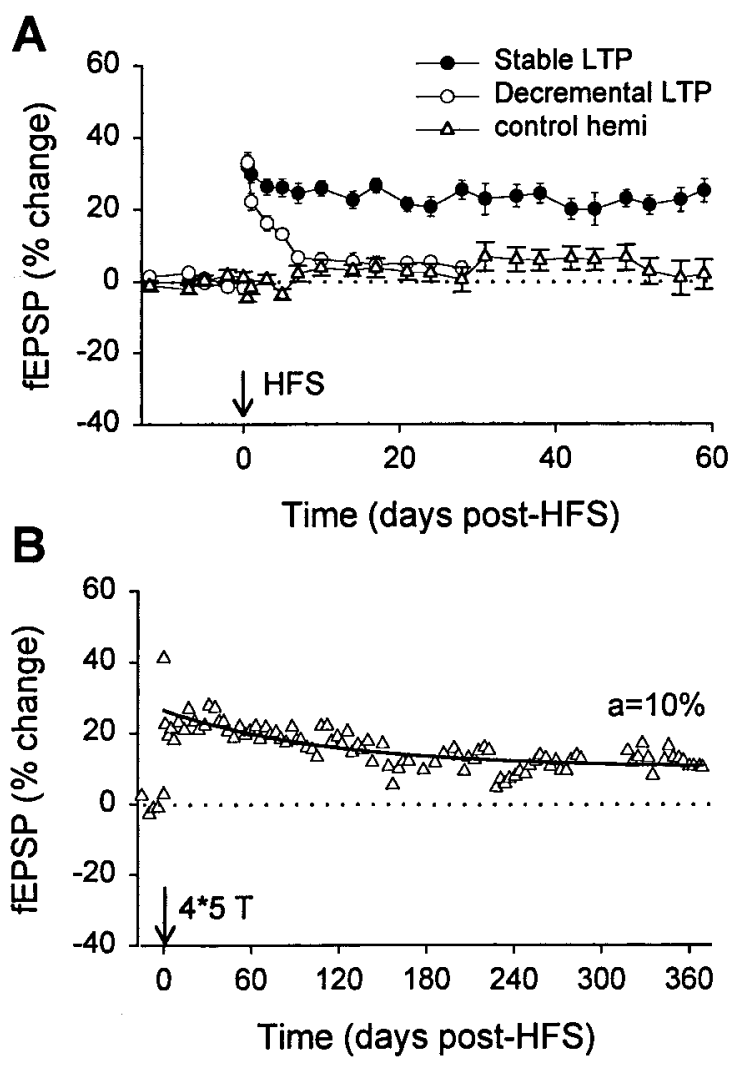

Figure 2. Maintenance of stable LTP. A, Summary LTP persistence plot for animals receiving 20 or more stimulus trains and exhibiting $>15 \%$ LTP, divided into two groups on the basis of whether the criteria for stable LTP were met $(\bullet, n=12)$ or not (i.e., LTP was decremental) $(\bigcirc$, $n=9)$. Data represent mean \pm SEM, corrected for control pathway changes. The available control hemisphere data, combined across the two groups, are also plotted $(\triangle ; n=9)$. Note the relative stability of the control hemisphere recordings. $B$, Stable LTP lasting 1 year post-HFS for an individual animal. Data are from the tetanized hemisphere, corrected for control pathway values that declined by $\sim 5 \%$ during the recording period. Solid curve is the fitted negative exponential function, with asymptote $(a)$ of $10 \%$ LTP and decay time constant of $127 \mathrm{~d}$.

animals showing $\geq 15 \%$ initial LTP, so that there was a nearly identical degree of LTP measured 60 min post-HFS for the two groups (stable LTP: $30 \pm 3 \%, n=12$; decremental LTP: $31 \pm$ $3 \%, n=9)$. Despite the concordance of LTP induction, the differential maintenance of LTP is striking. The average asymptote from the fitted exponential functions for the stable LTP group was $21 \pm 3 \%$ LTP, compared with $0.4 \pm 1 \%$ LTP for the decremental LTP group (Fig. $2 A$ ). For the stable LTP group, the average period of time post-HFS over which recordings were made before the recordings were terminated was $68 \mathrm{~d}$, with a range of 42-119 d. Importantly, recordings made in the control hemispheres over the same period of time remained stable, indicating that neither the stable nor decremental LTP was caused by chronic changes in animal physiology, movement, hormonal status, age, or similar whole-animal variables (Fig. $2 A)(n=9$; collapsed across type of LTP).

In one animal, the recordings were extended from the $119 \mathrm{~d}$ used in the above analysis to 1 year post-HFS (369 d) (Fig. 2B). The asymptote for the fitted exponential function fell only slightly during this time, from $13 \%$ for the $119 \mathrm{~d}$ function to $10 \%$ for the $369 \mathrm{~d}$ function, illustrating that LTP in perforant path-dentate gyrus synapses can be remarkably enduring, extending across a
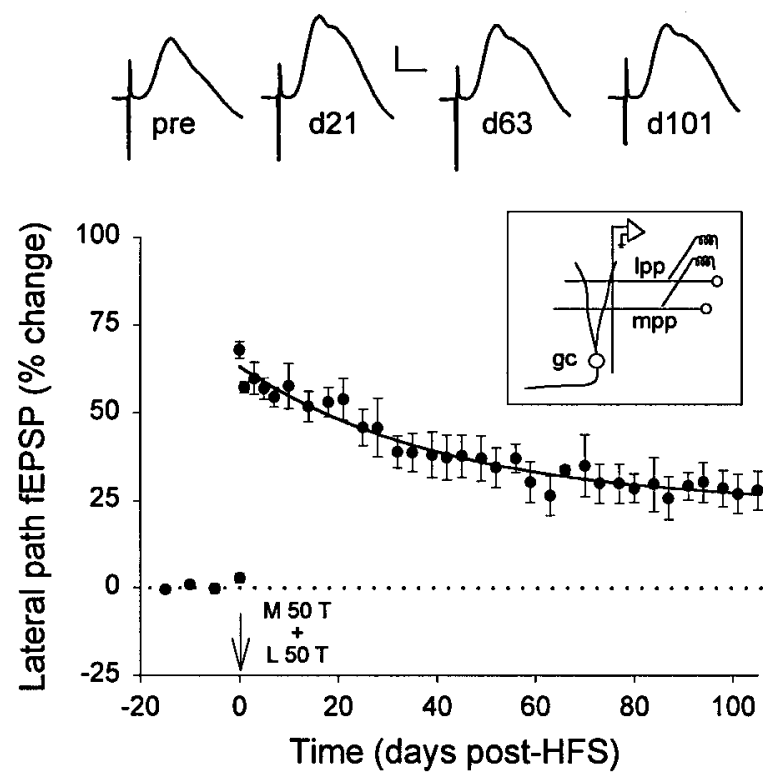

Figure 3. Induction of stable LTP in the lateral perforant path. Plotted are the average lateral path data for three animals that showed stable LTP after 50T HFS, simultaneously to both medial and lateral paths (arrow). Data have not been corrected because no control hemisphere recordings were made. Solid curve is the fitted negative exponential function for the average plot, with a time constant of $42 \mathrm{~d}$ and an asymptote of $24 \%$ LTP (a value very similar to the $21 \%$ asymptote obtained by averaging the asymptotes obtained for each individual animal). A fourth animal exhibited long-lasting but decremental LTP (data not shown). Waveforms are lateral path response averages of 30 sweeps taken just before tetanization ( pre), and on days $21(d 21), 63(d 63)$, and 101 (d101) post-HFS, for a representative animal. Calibration: $2 \mathrm{mV}, 5 \mathrm{msec}$. Inset diagram depicts the placement of stimulating electrodes separately in the lateral (lpp) and medial ( $m p p)$ perforant paths, plus a recording electrode in the dentate hilus, below the granule cell $(g c)$ layer of the dorsal blade.

significant portion of a rodent's lifespan. The slight reduction in the fitted asymptote, however, may be indicative of a slow decay of the LTP over time.

\section{Induction of stable LTP in lateral perforant path synapses}

Up to this point, the experiments involved electrical stimulation in the angular bundle that activated a mixture of medial and lateral path fibers, but principally those from the medial perforant path as determined by the electrophysiological properties of the responses. To determine whether lateral path synapses can also exhibit stable LTP, we implanted animals with separate stimulating electrodes in the medial and lateral aspects of the angular bundle. This allowed us to independently activate the lateral path before and after HFS (Abraham et al., 1994). During tetanization, the medial and lateral paths were coactivated using the 50T protocol to ensure a robust initial LTP in the lateral path, because we have found previously that the lateral path exhibits a relatively modest, rapidly decaying LTP when activated alone (Abraham et al., 1994). Using the combined tetanization protocol, we observed stable LTP lasting $>100 \mathrm{~d}$ in three of four animals, with an average exponential asymptote of $21 \pm 4 \%$ in the three animals showing stable LTP (Fig. 3).

\section{Sensitivity of stable LTP to variables on the day of tetanization}

It was curious that $50 \mathrm{HFS}$ trains (activating primarily medial path fibers) induced stable LTP in the present experiments, 

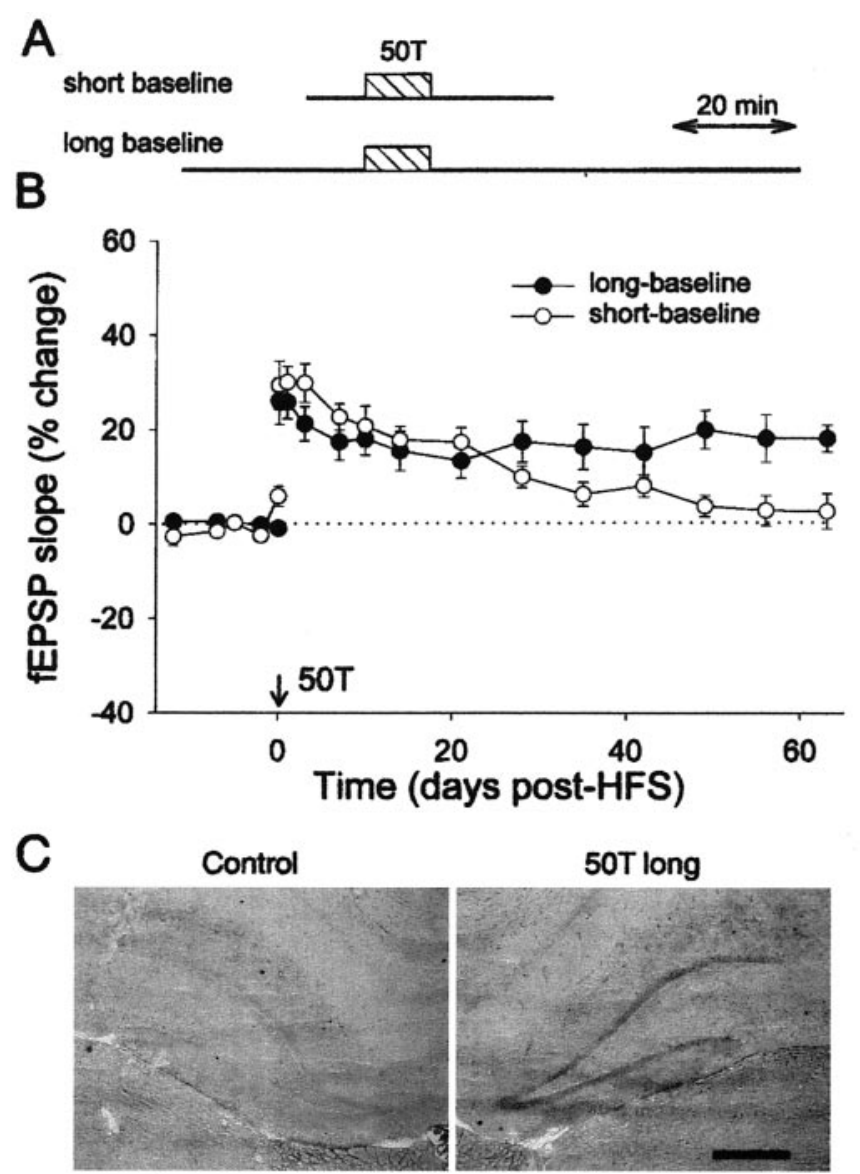

Figure 4. Summary LTP persistence plots for animals receiving 50T. A, Diagram of the timing of test-pulse recording periods (and time spent in the recording chamber) and the delivery of 50T HFS for the short- and long-baseline conditions. The 50T HFS took $10 \mathrm{~min}$ to complete. $B$, Animals given 50T with the long-baseline protocol (30 and $60 \mathrm{~min}$ test-pulse periods before and after LTP, respectively; $n=12$ ) generally showed stable LTP. Animals given short test-pulse periods of 10 and 20 min, respectively, showed decremental LTP for the same tetanization protocol $(n=9)$. The plotted data for the latter group (mean \pm SEM; corrected for control pathway values) represent a combination of three animals studied in the present experiment plus six animals that received the identical protocol and were reported by Abraham et al. (1995). The maintenance of LTP was statistically different between the long- and short-baseline groups (see Results). $C$, pCREB immunoreactivity in the dentate granule layer at $2 \mathrm{hr}$ after the 50 train long-baseline protocol, compared with the immunoreactivity found in the nontetanized hemisphere of the same animal. Scale bar, $0.5 \mathrm{~mm}$.

whereas we had routinely observed only decremental LTP using this same paradigm in previous studies (Abraham et al., 1993, 1995). Careful analysis of the relevant stimulus protocols revealed only one significant methodological difference. In the present experiments, recordings to test pulse stimulation were made for $30 \mathrm{~min}$ before and $60 \mathrm{~min}$ after the HFS, whereas previously we had used only $10 \mathrm{~min}$ and 20-30 min baseline recording periods, respectively (Fig. $4 A$ ). All of the tetanization parameters were otherwise identical. Indeed, it is noteworthy that many other previous studies using relatively short baselines also found dentate LTP to be decremental (Racine et al., 1983; Bloch and Laroche, 1985; de Jonge and Racine, 1985). To confirm that the length of the baseline recordings on the day of tetanization was a crucial protocol difference, three additional animals were given the short-baseline 50T paradigm. All three animals showed decremental LTP, with an average time constant of $32 \mathrm{~d}$, well within the range of our published findings for this protocol (Abraham and Otani, 1991; Abraham et al., 1993, 1995). The present data and the data from Abraham et al. (1995) are shown in combined form in Figure $4 B$ to illustrate the difference in LTP stability between the two types of 50T protocols. A two-way ANOVA with repeated measures on one factor revealed that there was a significant time $\times$ group interaction $\left(F_{(9,171)}=4.55\right.$; $p<0.001)$ and thus a faster decay over time for the short-baseline condition. This conclusion is supported by the fact that the frequency of occurrence of stable LTP for the short-baseline treatment (1 of 9 animals) was significantly less than that observed with the longer baseline protocol ( 8 of 12 animals; $\chi^{2}=$ 6.48; $p<0.05)$, although the levels of initial LTP induction were virtually identical. We tentatively interpret this difference in results as arising from the difference in the time of animal handling and transfer between the recording chamber and home cage, relative to the time of HFS. Although these procedures were highly practiced, the minor stress or stimulus during such handling close to the time of LTP induction in the short-baseline condition may have interfered with LTP stability. This has been shown previously for LTP in area CA1 (Xu et al., 1998; ManahanVaughan and Braunewell, 1999). We cannot rule out the possibility, however, that the difference in the numbers of test pulses delivered before and after the HFS may have contributed to the group differences in LTP stability.

\section{Relation of stable LTP to CREB phosphorylation}

There is considerable evidence across a number of species that the persistence of LTP and the establishment of long-term memory are linked by their dependence on the phosphorylation of the transcription factor CREB at serine-133 (Bourtchuladze et al., 1994; Yin et al., 1995; Schulz et al., 1999; Davis et al., 2000). To investigate whether the protocol that is efficient at inducing stable LTP is also distinguished by its ability to induce CREB phosphorylation (pCREB), new groups of animals, treated identically as above for the study of LTP persistence, were killed at 2 or $4 \mathrm{hr}$ after the two 50T protocols and processed for pCREB immunohistochemistry using a ser133-pCREB antibody. Delivery of the long-baseline 50T protocol produced a reliable increase in pCREB immunoreactivity relative to the control nontetanized hemisphere at $2 \mathrm{hr}$ post-HFS (three of three animals) (Fig. 4C). Quantitative image analysis revealed this effect to be significantly greater than the response following the short-baseline 50T protocol (long baseline: $23 \pm 8 \%$; short baseline: $-1 \pm 6 \%$; MannWhitney $U$ test; $p=0.05$ ). However, the effect was transient because there was no difference between groups in pCREB immunoreactivity at $4 \mathrm{hr}$ post-HFS ( $n=4$ both groups; MannWhitney $U$ test; $p=0.1$; data not shown). Administration of the NMDA receptor antagonist $(R S)$-3-(2-carboxypiperazin-4-yl)propyl-1-phosphonic acid (CPP; $10 \mathrm{mg} / \mathrm{kg}$, i.p.) blocked the induction of LTP by the 50T long-baseline protocol $(5 \pm 2 \% ; n=$ $4 ; p>0.1$ ), demonstrating the NMDA receptor dependence of stable LTP. CPP also significantly reduced the increase in pCREB immunoreactivity relative to the non-CPP-treated animals (Mann-Whitney $U$ test; $p=0.02$ ), although a small but statistically significant increase in immunoreactivity still occurred relative to the control hemisphere in the CPP-treated animals $(10 \pm 2 \% ; n=4$; paired $t$ test; $p<0.05)$. 

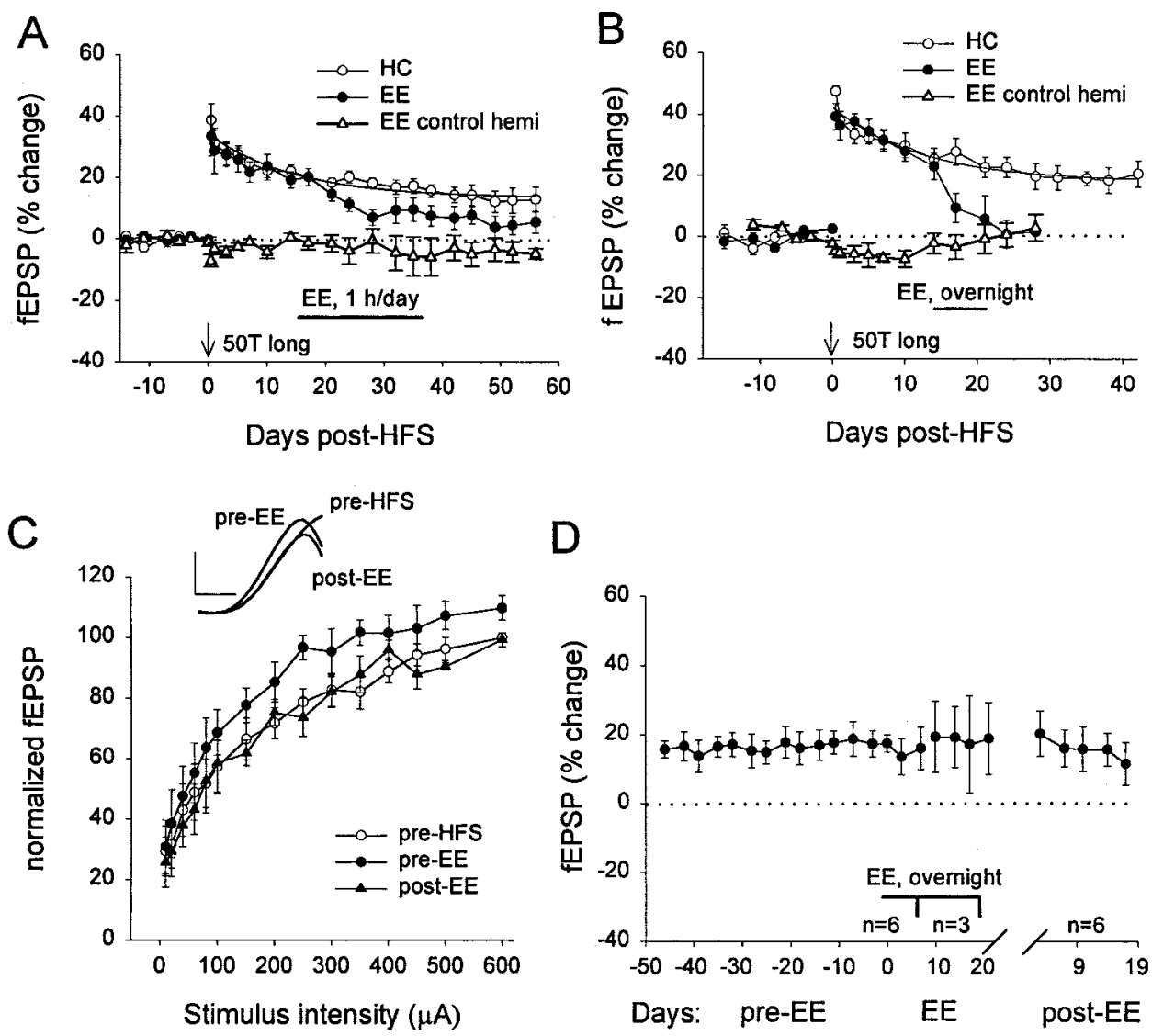
input-output curves for a single animal in the EE group. Illustrated are the rising phase of the fEPSP before population spike onset for a single animal. Calibration: $3 \mathrm{mV}, 0.5 \mathrm{msec}$. $D$, Overnight EE exposure failed to reverse LTP when given $\sim 90 \mathrm{~d}$ post-HFS (range $85-103 \mathrm{~d} ; n=6$ ). For convenience, data (corrected for control hemisphere changes) have been aligned for each animal relative to the start and finish of EE exposure. All six animals received EE exposure for 1 week, and three of these animals were given EE exposure for 2 further weeks. LTP for all six animals was monitored for a further $17 \mathrm{~d}$ after EE treatment.

\section{Reversal of stable LTP by repeated exposure to an enriched environment}

The stability of hippocampal LTP demonstrated above is remarkable given the classical view of the hippocampus as a temporary memory store (Marr, 1971; Milner, 1989; Squire, 1992). The stability of LTP may reflect, however, the fact that in our experiments the animals lived in an impoverished environment (single housing in small cages) without the opportunity for additional learning experiences that could compete or interfere with the newly established changes in synaptic weights, as may occur for animals living in a more naturalistic and changeable environment. To test this possibility, two groups of five animals were matched for standard perforant-path LTP induction and persistence over $14 \mathrm{~d}$ after long-baseline 50T HFS, using the criteria that the initial LTP was $\geq 15 \%$, the asymptote of the fitted negative exponential function over $14 \mathrm{~d}$ was $>10 \%$, and the LTP value on day 14 post-HFS was $\geq 10 \%$. One group (EE) was then placed in an enriched environment for $1 \mathrm{hr} / \mathrm{d}$ for 3 weeks. The novel environment provided the animals with many sources of information and opportunities for learning, including extra handling, a novel room, a novel and larger holding box, novel objects, social interaction, and increased motor activity. The animals in the other group were kept in their home cage (HC), except when tested electrophysiologically. As shown in Figure $5 A$, the $\mathrm{HC}$ group exhibited the expected development of stable LTP (average asymptote $=13 \%$ LTP). In contrast, the LTP in the EE group
Figure 5. Time-dependent reversal of stable LTP by exposure to enriched environments. A, Comparison of LTP maintenance for two groups: home cage controls $(H C ; n=5)$ and animals receiving $\mathrm{EE}$ exposure for $1 \mathrm{hr} / \mathrm{d}$ for 3 weeks $(E E ; n=$ $5)$. The EE group showed a lasting reversal of LTP. Data are corrected for changes in the control hemisphere that have also been plotted for the EE group $(n=4)$. Smooth curve is the fitted negative exponential function to the $\mathrm{HC}$ data; asymptote $=13 \%$ LTP. $B$, Exposure of a separate EE group $(n=5)$ to the enriched environment overnight for $7 \mathrm{~d}$ led to a more rapid and robust reversal of LTP maintenance, compared with a new $\mathrm{HC}$ group $(n=6)$. Because of equipment malfunction, recordings were not made in two EE animals beyond $3 \mathrm{~d}$ after EE treatment. Data are corrected for changes in the control hemisphere that are also plotted for the EE group $(n=5)$. Smooth curve is the fitted negative exponential function to the HC data; asymptote $=18 \%$ LTP. $C$, Input-output curves for the EE group presented in $B$. fEPSP slopes were measured across 15 stimulus strengths $(10-600 \mu \mathrm{A})$ and expressed as a percentage of the maximal response obtained pre-HFS. The data (uncorrected) were obtained at three time points: pre$H F S, 14 \mathrm{~d}$ post-HFS (pre-EE), and $21 \mathrm{~d}$ post-HFS (post-EE). EE exposure significantly reversed LTP across all stimulus strengths $\left(F_{(2,6)}=7.30 ; p<0.05\right)$. Inset waveforms are averages of 10 responses recorded during the same sessions as the range $85-103 \mathrm{~d} ; n=6$ ). For convenience,
and finish of EE exposure. All six animals LTP for all six animals was monitored for

gradually declined over the period of enrichment and remained significantly below the level of the $\mathrm{HC}$ group even after exposure to the novel environment was terminated (two-way ANOVA with repeated measures over days $7-45$ post-HFS; group $\times$ time interaction $\left.F_{(11,88)}=3.46 ; p<0.001\right)$. The response decay represents a depotentiation effect because it was specific to the potentiated hemisphere in the EE group. The responses in the control hemispheres of the same animals were little affected (Fig. $5 A$ ), apart from transient elevations of the population spike (data not shown).

In an attempt to induce a more robust LTP reversal, the "dose" of environmental enrichment was increased by giving a new set of animals overnight access $(14 \mathrm{hr} / \mathrm{d})$ to the enriched environment for a $7 \mathrm{~d}$ period, again beginning $14 \mathrm{~d}$ post-HFS. Indeed, this treatment produced a more rapid and dramatic reversal of LTP $(n=5)$, as compared with a new matched set of HC controls $(n=$ 6 ), which again showed stable LTP with an average asymptote of 18\% LTP (Fig. 5B). The two groups showed statistically different levels of LTP, as indicated by a repeated-measures ANOVA over days 7-24 post-HFS (group $\times$ time interaction $F_{(5,40)}=5.87 ; p<$ $0.001)$. Input-output analysis revealed that the reversal of LTP occurred equivalently across a range of stimulus strengths (Fig. $5 C$ ). Finally, to determine whether the reversibility of LTP was time-dependent, six animals showing stable LTP for $\sim 90 \mathrm{~d}$ (range $=85-103 \mathrm{~d} ; n=6$ ) were subsequently given overnight exposure to the enriched environment for either $7(n=3)$ or $21 \mathrm{~d}$ 
$(n=3)$. Although this treatment increased the variability of the responses somewhat, it did not change the average degree of LTP expressed either during or after the period of exposure to the enriched environment (average LTP just before EE: $16 \pm 6 \%$; just after EE: $20 \pm 7 \%$ ) (Fig. $5 D$ ).

\section{DISCUSSION}

The hippocampus and associated medial temporal lobe structures are believed by many theorists to serve as a holding store for mnemonic information, while contributing to the gradual integration of the newly learned information into the memory structures of the neocortex (Teyler and Discenna, 1985; McClelland et al., 1995; Eichenbaum et al., 1996; Rolls, 1996). Accordingly, it has been suggested that learning-associated synaptic plasticity should be rapidly but transiently induced in the hippocampus, whereas plasticity in the neocortex would require repeated activation episodes, with small but stable increments of synaptic change in response to each episode (Milner, 1989; McClelland et al., 1995). On the whole, previous data describing the decremental nature of LTP in the dentate gyrus region of hippocampus have been in accord with this view (Racine et al., 1983; Abraham and Otani, 1991). However, Staubli and Lynch (1987) reported that stable (nondecremental) LTP lasting 1-5 weeks could be induced by theta-burst stimulation in area CA1, and Bliss and GardnerMedwin (1973) reported a case of stable maintenance of LTP in rabbit dentate gyrus over many weeks. Here we have revisited this issue for the dentate gyrus and found that this hippocampal subregion indeed has the capacity for stable LTP. Thus, we were able to observe stable LTP lasting months for both medial and lateral perforant path synapses, and in one case lasting up to 1 year, a significant proportion of a rodent's lifespan. Importantly, we used exponential curve-fits for data from individual animals to provide a quantitative measure of stability. This proved useful, in part, because the LTP often underwent a period of slow decline before reaching its apparent asymptotic level. The present demonstration of very long-term stability of LTP indicates that LTP does indeed have the capacity, after a single induction episode, to underlie memory storage across months or more. Although it is vital to know whether LTP has this capacity, it is nonetheless unknown whether real world memory mechanisms require such stable plasticity because, for example, occasional periods of memory retrieval and rehearsal could restrengthen any underlying synaptic plasticity before it decays completely.

What are the implications of stable LTP for our understanding of hippocampal function? Long-term maintenance of LTP appears to challenge the conventional wisdom that the hippocampus is a temporary memory store. It is consistent, however, with recent controversial suggestions from clinical cases (showing prolonged retrograde amnesia after hippocampal damage) that this brain region may be involved more directly in long-term memory storage than previously supposed (Nadel and Moscovitch, 1997; Cipolotti et al., 2000). In rats, the long-term storage of information in the hippocampus might support both the establishment (Kentros et al., 1998) and the long-term stability of the place fields of hippocampal neurons (Thompson and Best, 1990; Lever et al., 2002) or aid the generation and updating of stable spatial reference frames that may be important for animal navigation (McNaughton et al., 1996). It may be, however, that such interpretations are valid only for animals living in a stimulus-poor environment, because we observed that otherwise stable LTP rapidly decayed after repeated novel experiences. Thus for ani- mals living in a naturalistic and changing environment, LTP (and perhaps memory storage) in the hippocampus may normally be short-lasting, in accord with the above-mentioned prevailing views of hippocampal function.

The reversal of LTP could be attributable to any number of behavioral variables associated with experiences in the enriched environment. It seems likely, however, that extra information processing in the dentate gyrus was somehow responsible for resetting the weights of the perforant path synapses. Such resetting was presumably caused by a homosynaptic long-term depression or depotentiation effect, because it occurred for the potentiated synaptic responses but not for the control responses recorded in the opposite hemisphere of the same animals. Whatever the mechanism, the reversal of LTP may represent a synaptic mechanism underlying the psychological phenomenon of retrograde interference, whereby newly learned information interferes with the retrieval of previously learned information.

Our findings differ from previous studies of LTP reversal by novel experience, which were conducted in CA1 and had a narrow time window of effect spanning only several hours after LTP induction (Xu et al., 1998; Manahan-Vaughan and Braunewell, 1999). In contrast, we observed that repeated exposure to enriched environments reversed LTP even when commencing $14 \mathrm{~d}$ after induction. Thus, the mechanism of LTP disruption may differ between the two sets of studies. Nonetheless, we did find that stable LTP had a consolidation phase, because it eventually became resistant to reversal by enrichment by 3 months after induction. This finding is thus consistent with the recent observation that hippocampus-dependent memory also consolidates across weeks after initial training (Shimizu et al., 2000). This prolonged consolidation of memory was dependent on NMDA receptor activation during the consolidation period. Whether LTP consolidation is also NMDA receptor dependent remains to be investigated.

Previous experiments using post-training novel environments have produced conflicting effects on the retention of hippocampusdependent memory. On the one hand, exposure to a novel open field inhibited retention of inhibitory avoidance performance acquired $1 \mathrm{hr}$ previously (Viola et al., 2000). On the other hand, post-training exposure to an enriched environment for 2 weeks facilitated retention of contextual fear conditioning (Feng et al., 2001). The former study is consistent with the studies showing a particular sensitivity of LTP to reversal early after its induction (Xu et al., 1998; Manahan-Vaughan and Braunewell, 1999). The latter study, however, appears to contrast directly with the present one, because we observed that new behavioral experiences caused a reversal of a synaptic memory trace (i.e., LTP). These findings of Feng et al. (2001) and the present study are not necessarily contradictory, however, and we propose the following solution to the apparent paradox. Exposure to an enriched environment facilitates memory retention (Feng et al., 2001) because it facilitates longerterm storage in the neocortex. At the same time, it promotes erasure of the memory trace in the hippocampus, as exemplified by the reversal of LTP in our experiments, to facilitate new learning. This hypothesis can be tested experimentally, because it predicts that the retrograde amnesia gradient resulting from hippocampal damage will be shortened for animals exposed to an enriched environment after training, because of more rapid consolidation in other structures.

In the present experiments, the induction of stable LTP was associated with the phosphorylation of CREB, which may play a 
role in signaling gene expression critical for LTP maintenance. Our findings thus support previous studies demonstrating that hippocampal LTP protocols can cause increases in PCREB and CRE-mediated gene expression that are dependent only partially on NMDA receptor activation (Impey et al., 1996) and are associated with the late phase of LTP (Bourtchuladze et al., 1994; Schulz et al., 1999; Davis et al., 2000). We have extended these findings for dentate LTP, however, by showing that pCREB is particularly associated with protocols that induce stable LTP lasting months, whereas LTP that is long-lasting but nonetheless decremental can occur independently of raised pCREB levels (Walton et al., 1999). We tentatively attribute the failure of the short-baseline HFS protocol to readily induce either pCREB or stable LTP to the behavioral disruption accompanying the removal of the animals from the recording chamber to their home cage. This is consistent with the demonstration that both traininginduced pCREB expression in the hippocampus and long-term memory formation are inhibited in a time-dependent manner by exposure of the animals to a mild stressor (i.e., a novel environment) after the learning experience (Viola et al., 2000). It should be noted, however, that the duration of LTP maintenance is likely regulated by a host of transcription factors in addition to $\mathrm{PCREB}$, as well as by other genes coding for cytoplasmic proteins (Abraham et al., 1993; Jones et al., 2001), and the persistence of LTP can vary dramatically (Abraham and Otani, 1991; present results). Thus the late phase of LTP in vivo may not be a unitary phenomenon and may depend instead on the complement and pattern of expression of the different signaling molecules induced by a stimulation protocol or learning experience.

In summary, we have addressed one of the key issues regarding LTP, namely, whether it can in principle be maintained long enough to serve as a mechanism underlying stable long-term memory. Our data indicate that hippocampal LTP in adult animals does indeed have this capacity. Furthermore, if the LTP survives intact over the first few weeks after its induction, it then becomes resistant to further change and in principle may contribute either to very long-term memory storage or to a very longterm change in the way that new information is processed during subsequent experience. This may apply only to animals living in stimulus-poor environments, however. Under naturalistic conditions, LTP in the hippocampus appears to be readily overwritten as a result of new experiences, in accordance with conventional theories of hippocampal function. It remains to be investigated whether LTP is both stable and resistant to reversal in other structures, such as the neocortex, that are believed to be responsible for permanent memory storage.

\section{REFERENCES}

Abraham WC, Otani S (1991) Macromolecules and the maintenance of long-term potentiation. In: Kindling and synaptic plasticity (Morrell F, ed), pp 92-109. Boston: Birkhäuser.

Abraham WC, Demmer J, Richardson C, Williams J, Lawlor P, Mason SE, Tate WP, Dragunow M (1993) Correlations between immediate early gene induction and the persistence of long-term potentiation. Neuroscience 56:717-727.

Abraham WC, Christie BR, Logan B, Lawlor P, Dragunow M (1994) Immediate early gene expression associated with the persistence of heterosynaptic long-term depression in the hippocampus. Proc Natl Acad Sci USA 91:10049-10053.

Abraham WC, Mason-Parker SE, Williams J, Dragunow M (1995) Analysis of the decremental nature of LTP in the dentate gyrus. Mol Brain Res 30:367-372.
Abraham WC, Logan BL, Greenwood J, Dragunow M (2001) CREB phosphorylation-associated stable LTP lasting months in the hippocampus. Proc Int Australas Winter Conf Brain Res 19:41.

Bliss TVP, Gardner-Medwin AR (1973) Long-lasting potentiation of synaptic transmission in the dentate area of the unanaesthetized rabbit following stimulation of the perforant path. J Physiol (Lond) 232:357-374.

Bloch V, Laroche S (1985) Enhancement of long-term potentiation in the rat dentate gyrus by post-trial stimulation of the reticular formation. J Physiol (Lond) 360:215-231.

Bourtchuladze R, Frenguelli B, Blendy J, Cioffi D, Schutz G, Silva AJ (1994) Deficient long-term memory in mice with a targeted mutation of the cAMP-responsive element-binding protein. Cell 79:59-68.

Butterworth NJ, Dragunow M (1996) Medial septal cholinergic neurons express c-Jun but do not undergo DNA fragmentation after fornixfimbria transections. Mol Brain Res 43:1-12.

Cipolotti L, Shallice T, Chan D, Fox N, Scahill R, Harrison G, Stevens J, Rudge P (2000) Long-term retrograde amnesia: the crucial role of the hippocampus. Neuropsychologia 39:151-172.

Davis S, Vanhoutte P, Pages C, Caboche J, Laroche S (2000) The MAPK/ERK cascade targets both Elk-1 and cAMP response elementbinding protein to control long-term potentiation-dependent gene expression in the dentate gyrus in vivo. J Neurosci 15:4563-4572.

de Jonge M, Racine RJ (1985) The effects of repeated induction of long-term potentiation in the dentate gyrus. Brain Res 328:181-185.

Eichenbaum H, Schoenbaum G, Young B, Bunsey M (1996) Functional organization of the hippocampal memory system. Proc Natl Acad Sci USA 93:13500-13507.

Feng R, Rampon C, Tang Y-P, Shrom D, Jin J, Kyin M, Sopher B, Martin GM, Kim S-H, Langdon R, Sisodia SS, Tsien JZ (2001) Deficient neurogenesis in forebrain-specific presenilin-1 knockout mice is associated with reduced clearance of hippocampal memory traces. Neuron 32:911-925.

Huang Y-Y, Kandel ER (1994) Recruitment of long-lasting and proteinkinase A-dependent long-term potentiation in the CA1 region of hippocampus requires repeated tetanization. Learn Mem 1:74-82.

Impey S, Mark M, Villacres EC, Poser S, Chavkin C, Storm DR (1996) Induction of CRE-mediated gene expression by stimuli that generate long-lasting LTP in area CA1 of the hippocampus. Neuron 16:973-982.

Irvine GI, Abraham WC (2001) Failure of an enriched environment to induce long-term potentiation in freely moving rats. Proc Int Australas Winter Conf Brain Res 19:53.

Jones MW, Errington ML, French PJ, Fine A, Bliss TVP, Garel S, Charnay P, Bozon B, Laroche S, Davis S (2001) A requirement for the immediate early gene Zif268 in the expression of late LTP and longterm memories. Nat Neurosci 4:289-296.

Kentros C, Hargreaves E, Hawkins RD, Kandel ER, Shapiro M, Muller RU (1998) Abolition of long-term stability of new hippocampal place cell maps by NMDA receptor blockade. Science 280:2121-2126.

Lever C, Wills T, Cacucci G, Burgess N, O'Keefe J (2002) Long-term plasticity in hippocampal place-cell representation of environmental geometry. Nature 416:90-94.

Manahan-Vaughan D, Braunewell KH (1999) Novelty acquisition is associated with induction of hippocampal long-term depression. Proc Natl Acad Sci USA 96:8739-8744.

Marr D (1971) Simple memory: a theory for archicortex. Philos Trans R Soc Lond B Biol Sci 262:23-81.

McClelland JL, McNaughton BL, O'Reilly RC (1995) Why there are complementary learning systems in the hippocampus and neocortex: insights from the successes and failures of connectionist models of learning and memory. Psychol Rev 102:419-457.

McNaughton BL, Barnes CA Gerrard JL, Gothard K, Jung MW, Knierim JJ, Kudrimoti H, Qin Y, Skaggs WE, Suster M, Weaver KL (1996) Deciphering the hippocampal polyglot: the hippocampus as a path integration system. J Exp Biol 199:173-185.

Milner P (1989) A cell assembly theory of hippocampal amnesia. Neuropsychologia 27:23-30.

Nadel L, Moscovitch M (1997) Memory consolidation, retrograde amnesia and the hippocampal complex. Curr Opin Neurobiol 7:217-227.

Racine RJ, Milgram NW, Hafner S (1983) Long-term potentiation phenomena in the rat limbic forebrain. Brain Res 260:217-231.

Rolls ET (1996) A theory of hippocampal function in memory. Hippocampus 6:601-620.

Schulz S, Siemer H, Krug M, Höllt V (1999) Direct evidence for biphasic cAMP responsive element-binding protein phosphorylation during long-term potentiation in the rat dentate gyrus in vivo. J Neurosci 19:5683-5692.

Shimizu E, Tang Y-P, Rampon C, Tsien JZ (2000) NMDA receptordependent synaptic reinforcement as a crucial process for memory consolidation. Science 290:1170-1174.

Squire LR (1992) Memory and the hippocampus: a synthesis from findings with rats, monkeys, and humans. Psychol Rev 99:195-231.

Staubli U, Lynch G (1987) Stable hippocampal long-term potentiation elicited by "theta" pattern stimulation. Brain Res 435:227-234. 
Teyler TJ, Discenna P (1985) The role of hippocampus in memory: a hypothesis. Neurosci Biobehav Rev 9:377-390.

Thompson LT, Best PJ (1990) Long-term stability of the place-field activity of single units recorded from the dorsal hippocampus of freely behaving rats. Brain Res 509:299-308.

Viola HE, Furman M, Izquierdo LAI, Alonso M, Barros DM, De Souza MM, Izquierdo I, Medina JH (2000) Phosphorylated cAMP response element-binding protein as a molecular marker of memory processing in rat hippocampus: effect of novelty. J Neurosci 20:RC112(1-5).
Walton M, Henderson C, Mason-Parker S, Lawlor P, Abraham WC, Bilkey D, Dragunow M (1999) Immediate early gene transcription and synaptic modulation. J Neurosci Res 58:96-106.

Xu L, Anwyl R, Rowan MJ (1998) Spatial exploration induced a persistent reversal of long-term potentiation in rat hippocampus. Nature 394:891-894.

Yin JCP, Del Vecchio M, Tully T (1995) CREB as a memory modulator: induced expression of a dCREB2 activator isoform enhances long-term memory in Drosophila. Cell 81:107-115. 